\title{
Efectos de tratamientos térmicos post-alcalinos sobre las propiedades superficiales de titanio para implantes dentales con diferentes recubrimientos
}

\section{Effects of heat treatment on surface properties of different coatings with alkali treatment on titanium for dental implants}

\author{
Tatiana Ekkert ${ }^{1}$, Adriana Lemos ${ }^{1}$, Kyung Won Kang ${ }^{1}$, Florencia Gatti ${ }^{1}$ \\ Carlos Llorente ${ }^{1,2}$, Pablo Bilmes ${ }^{1}$
}

\footnotetext{
${ }^{1}$ Laboratorio de Investigaciones de Metalurgia Física (LIMF), Facultad de Ingeniería, Universidad Nacional de La Plata, La Plata CP: 1900, Argentina.

${ }^{2}$ Comisión de Investigaciones Científicas de la Provincia de Buenos Aires - CICPBA.

e-mail: tatiana.ekkert@ing.unlp.edu.ar
}

\section{RESUMEN}

El titanio es ampliamente utilizado como implante dental dado que es bioinerte y osteointegrable. Sin embargo, dado que este material no puede inducir el crecimiento de hueso desde su superficie, es usual que se le realicen distintos tratamientos superficiales para desarrollar recubrimientos bioactivos. El tratamiento alcalino tiene por objetivo formar un gel de titanato de sodio amorfo, el cual se puede estabilizar, por medio de un tratamiento térmico posterior. Durante este último, el hidrogel se deshidrata y se densifica para formar una capa de titanato de sodio estable y parcialmente cristalizado. En el presente trabajo se analizaron los efectos producidos por diferentes tratamientos térmicos posteriores al alcalino, sobre superficies de titanio cp con tratamiento superficial de blastinizado y APQ (anodizado por plasma químico). Para cada caso se caracterizó la superficie con el fin de optimizar la condición de aplicación. Las temperaturas de los tratamientos térmicos fueron 400,600 y $800^{\circ} \mathrm{C}$, dado que en este rango el titanato varía su proporción de fase amorfa y cristalina y por ende sus propiedades. La evaluación de los resultados se realizó mediante ensayos de adhesión Rockwell C, medición del ángulo de contacto inicial por goniometría, difracción de rayos x, SBF y microscopia electrónica de barrido.

Caracterizadas las probetas post tratamiento térmico se pudo observar que, las muestras tratadas a $800{ }^{\circ} \mathrm{C}$ presentaron una estructura superficial cristalina, mala adhesión y no indujeron crecimiento de apatita sobre su superficie. No obstante presentaron una superficie hidrofilica.

Por otro lado, las muestras tratadas a 400 y $600{ }^{\circ} \mathrm{C}$ presentaron superficialmente una fase parcialmente cristalizada, con buena adherencia y buena hidrofilicidad. Mediante SEM-EDS, se observó sobre sus superficies la formación de una capa de apatita homogénea.

Palabras clave: titanio, blastinizado, anodizado por plasma químico, tratamiento alcalino, tratamiento térmico.

\section{ABSTRACT}

Titanium is widely used as dental implant since it is bio-inert and osseointegratable. However, provided that this material cannot induce bone growth from its surface, it is usual to carry out different surface treatments in order to develop bioactive coatings that increase both the initial rate of bone development and the biological anchorage of implant attachment to the host bone. The aim of the alkaline treatment is to form an amorphous gel of sodium titanate, which can be stabilized by means of a subsequent thermal treatment. During this, the hydro gel is dehydrated and thickened to form a layer of stable and partially crystallized sodium titanate. In the present work it was analyzed the effects produced by different thermal treatments after the alka- 
line one, on surfaces of $\mathrm{cp}$ titanium with surface treatment of blasting and MAO (micro-arc oxidation process). For each case the surface was characterized in order to enhance the application condition. The temperature of the thermal treatments was 400,600 and $800{ }^{\circ} \mathrm{C}$, since titanate varies its ratio of amorphous and crystalline phase in this range and therefore its properties. The assessment of results was carried out by means of Rockwell-C adhesion standard test, measurement of the initial contact angle by goniometry, X-ray diffraction and analytic scanning electronic microscopy.

Characterized post thermal treatment samples, it was observed that the samples treated to $800{ }^{\circ} \mathrm{C}$ presented a crystalline superficial structure, bad adherence and did not develop apatite growth on its surface. Nevertheless, they presented sufficiently surface hydrophilic.

On the contrary, the samples treated at 400 and $600^{\circ} \mathrm{C}$ presented superficially a partially crystallized phase, with good adhesion and good hydrophilicity. Through SEM-EDS, the formation of a homogeneous apatite layer was observed on their surfaces.

Keywords: Titanium, blasting, micro-arc oxidation, alkali treatment, heat treatment.

\section{INTRODUCCIÓN}

El titanio es ampliamente utilizado en odontología debido a sus excelentes características en cuanto a inercia química, resistencia mecánica con bajo módulo elástico, baja densidad y toxicidad, buena resistencia a la corrosión, y fundamentalmente por ser biocompatible y osteointegrable [1]. A nivel mundial, más de 1000 toneladas de titanio se implantan anualmente en seres humanos en forma de dispositivos biomédicos [2]. El titanio posee una elevada resistencia a la corrosión debido a la presencia de una fina capa de óxido de titanio de 2 a $7 \mathrm{~nm}$ de espesor $[3,4]$.

Sin embargo, debido a la incapacidad de este material de inducir el crecimiento de hueso directamente desde su superficie, se llevan a cabo distintos tratamientos superficiales, dependiendo de la aplicación específica. El objetivo es desarrollar un recubrimiento bioactivo que aumente tanto la velocidad inicial de desarrollo óseo como el anclaje biológico de fijación del implante al hueso.

En los últimos tiempos se han desarrollado una gran variedad de tratamientos superficiales que se realizan mediante modificaciones físicas y/o químicas. Uno de los métodos más tradicionales es el blastinizado, dónde se utilizan partículas de sílice y alúmina de distintos tamaños y formas [4]. Actualmente se han empezado a utilizar partículas biocompatibles de calcio y fósforo debido a que mejoran la interacción del implante con el tejido.

Dentro de los métodos más modernos, sobresalen aquellos que producen recubrimientos bioactivos como es el caso del APQ y los tratamientos termo químicos. El tratamiento de anodizado por plasma químico (APQ) [5], también conocido como proceso MAO (micro-arcoxidation), genera un recubrimiento de tipo cerámico que posee una elevada microdureza, adhesión al metal base y de alta resistencia al desgaste [6]. Este proceso se basa en la ruptura dieléctrica de una capa de óxido aislante en la superficie de un ánodo metálico en contacto con un electrolito adecuado. Durante el proceso aparecen chispas en la superficie del ánodo y desprendimiento de gas en forma de burbujas. El recubrimiento obtenido, que se compone de elementos del ánodo metálico y del electrolito, es una capa de óxido de titanio con $\mathrm{P}$ y $\mathrm{Ca}$, bioactiva, porosa, rugosa y firmemente adherida a la superficie. Estas características topográficas y composicionales dependen de los parámetros del tratamiento de APQ, de la composición del electrolito, densidad de corriente o voltaje aplicado y tiempo [7].

Por otro lado, dentro de los tratamientos termo químicos se destaca el tratamiento alcalino, que induce la formación de un hidrogel de titanato de sodio amorfo que favorece el crecimiento de apatita sobre la superficie del implante [8]. Además cumple la función de transformar las superficies bioinertes a bioactivas logrando mejorar en tiempo y forma el proceso de osteointegración [8,9]. El gel de titanato de sodio que se forma durante el tratamiento alcalino, se puede estabilizar por medio de un tratamiento térmico, como una capa de titanato de sodio denso y parcialmente cristalizado [10,11], cuyo propósito es la mejora de la adhesión y bioactividad del recubrimiento en la superficie del implante. Cabe aclarar que la formación de la apatita dependerá, entre otros factores, de la capacidad de migración de iones de sodio, por lo cual el grado de cristalización del hidrogel va a repercutir en los tiempos de nucleación de la apatita. En el presente trabajo se analizaron los efectos que producen diferentes tratamientos térmicos posteriores al alcalino, sobre superficies de titanio cp con tratamiento superficial de blastinizado y APQ (anodizado por plasma químico). Se evaluaron tres temperaturas de trabajo 400,600 y $800^{\circ} \mathrm{C}$ con el fin de hallar una temperatura óptima de aplicación.

Las superficies se caracterizaron mediante ensayos de ángulo de contacto, difracción de rayos x, SBF, adhesión y microscopia electrónica de barrido. Las muestras tratadas a $800^{\circ} \mathrm{C}$ revelaron una fase completa- 
mente cristalina, repercutiendo desfavorablemente en los resultados obtenidos en adhesión y en el crecimiento de la capa de apatita. Sin embargo las muestras tratadas a 400 y $600^{\circ} \mathrm{C}$ revelaron una fase parcialmente cristalizada y desarrollaron una superficie bioactiva que indujo el crecimiento de apatita sobre las mismas.

\section{MATERIALES Y MÉTODOS}

\subsection{Preparación de muestras}

El material de estudio consistió en probetas cilíndricas de Ti c.p ASTM Grado 4, con un diámetro de 9 mm y una altura de $4 \mathrm{~mm}$. A partir de las mismas se establecieron dos condiciones de análisis: probetas blastinizadas con partículas de calcio y fósforo y por otro lado, probetas blastinizadas con posterior anodizado por plasma químico (APQ) con un electrolito rico en Ca y $\mathrm{P}$ a base de fosfato de calcio anhidro (CaHPO4) y acetato de calcio $(\mathrm{Ca}(\mathrm{CH} 3 \mathrm{COO}) 2)$.

\subsection{Tratamiento alcalino}

Ambas condiciones de partida fueron sometidas a un tratamiento alcalino que consistió en sumergir las muestras en una solución de $\mathrm{NaOH} 10 \mathrm{M}$ durante 24 hs a una temperatura de $60^{\circ} \mathrm{C}$. El objetivo del tratamiento fue formar un gel de titanato de sodio sobre las superficies de las muestras.

\subsection{Tratamiento térmico}

El tratamiento térmico consistió en exponer las probetas post tratamiento alcalino a las temperaturas de interés para densificar el gel y mejorar la adhesión al sustrato. Las probetas se colocaron en un crisol y se llevaron durante 1 hora al horno a 400,600 y $800^{\circ} \mathrm{C}$.

\section{CARACTERIZACIÓN DE LAS SUPERFICIES}

\subsection{Adhesión Rockwell C}

Se realizaron tres indentaciones por muestra con una carga de $150 \mathrm{~kg}$ con el fin de evaluar la calidad y la resistencia adhesiva de la capa de titanato formada. Se utilizó un durómetro BSG DL 171 y un penetrador cónico de diamante con una punta de $200 \mu \mathrm{m}$ de diámetro. A continuación se observaron en el microscopio electrónico de barrido y la calidad de la adhesión del recubrimiento se comparó con la clasificación especificada por la norma VDI 3198[13].

\subsection{Angulo de contacto}

Se realizó un ensayo de mojabilidad mediante la determinación del ángulo de contacto inicial por goniometría. Se utilizó agua como líquido de prueba, con un goniómetro ramé-hart Model 500 (ramé-hart instrumentco.,USA).

\subsection{Difracción de rayos $X$}

Los estudios de difracción se realizaron con un equipo de rayos x para polvos cristalinos marca Panalytical, modelo Empyrean, con detector PixCel 3 de haz rasante con un ángulo de $1^{\circ}$ en un rango de barrido de 15 a $70^{\circ}$ con paso de $0,02^{\circ}$ y $1 \mathrm{seg} /$ paso. Se analizó el cambio de proporciones de fase amorfa a cristalina del recubrimiento a las distintas temperaturas de trabajo.

\subsection{SBF}

El análisis de la respuesta biológica de las muestras se realizó mediante un ensayo de SBF (Simulated Body Fluid). El mismo consistió en sumergir las muestras en la solución preparada según norma ISO/FDIS 233172012 [14] durante 7 días a una temperatura de $37^{\circ} \mathrm{C}$.

\subsection{SEM-EDS}

Las superficies luego de la inmersión en SBF fueron observadas mediante microscopia electrónica de barrido FEI QUANTA 200 y analizadas por espectroscopia dispersiva en energías (EDS) para determinar la relación 
$\mathrm{Ca} / \mathrm{P}$. El voltaje utilizado durante el ensayo fue de $20 \mathrm{kV}$ con un aumento de $100 \mathrm{x}$.

En la tabla 1 se presenta la nomenclatura utilizada en este trabajo para los diferentes tratamientos.

Tabla 1: Nomenclatura de las probetas de estudio.

\begin{tabular}{l|l}
\hline NOMBRE COMPLETO SEGUN TRATAMIENTO & ABREVIATURA \\
\hline Blasting+TratamientoAlcalino+Tratamiento Térmico a $400^{\circ} \mathrm{C}$ & BNaOH-T400 \\
\hline Blasting+TratamientoAlcalino+Tratamiento Térmico a $600{ }^{\circ} \mathrm{C}$ & BNaOH-T600 \\
\hline Blasting+TratamientoAlcalino+TratamientoTermico a $800^{\circ} \mathrm{C}$ & BNaOH-T800 \\
\hline Blasting+APQ+TratamientoAlcalino+Tratamiento Térmico a $400^{\circ} \mathrm{C}$ & BAPQNaOH- \\
\hline & BAPQNaOH- \\
\hline Blasting+APQ+TratamientoAlcalino+Tratamiento Térmico a $600{ }^{\circ} \mathrm{C}$ & T600 \\
\hline & BAPQNaOH- \\
Blasting+APQ+TratamientoAlcalino+Tratamiento Térmico a $800^{\circ} \mathrm{C}$ & T800 \\
\hline
\end{tabular}

\section{RESULTADOS}

\subsection{Angulo de Contacto}

Mediante el ensayo de medición de ángulo de contacto se caracterizó la hidrofilia de las superficies de las muestras tratadas termoquímicamente a 400,600 y $800^{\circ} \mathrm{C}$. Se realizaron tres mediciones por muestra. Tanto las muestras de blasting, como las de APQ con tratamiento alcalino y evaluadas a las tres temperaturas de trabajo presentaron un alto grado de hidrofilidad en sus superficies. En algunos casos, el equipo no pudo medir el ángulo ya que el proceso de mojado fue demasiado rápido. Los valores registrados durante el ensayo se presentan en la Tabla 1.

Tabla 2: Valores de ângulos de contacto de las muestras tratadas termicamente a diferentes temperaturas.

\begin{tabular}{l|c|c|c}
\hline \multicolumn{1}{c|}{ MUESTRA } & \multicolumn{3}{c}{ ANGULO DE CONTACTO () } \\
\hline BNaOH-T400 & 11 & 7 & Sin medición \\
\hline BNaOH-T600 & 7,1 & 8,6 & Sin medición \\
\hline BNaOH-T800 & 7,2 & 8,6 & Sin medición \\
\hline BAPQNaOH-T400 & 7 & 9 & Sin medición \\
\hline BAPQNaOH-T600 & 7 & 9 & Sin medición \\
\hline BAPQNaOH-T800 & 7 & 9 & 9 \\
\hline
\end{tabular}

La hidrofilia es un parámetro de suma importancia para la biocompatibilidad y la capacidad de osteointegración del implante. A su vez es crítica para el proceso de adhesión inicial de los componentes celulares y las proteínas, entre otras macromoléculas.

\subsection{Dureza}

La calidad y la resistencia adhesiva de la capa de titanato se evaluó mediante el ensayo de indentación Rockwell C. Para ello, se realizaron tres indentaciones con una carga de $150 \mathrm{~kg}$ en cada una de las muestras. Estas indentaciones se observaron mediante microscopía electrónica de barrido y la calidad de adhesión del recubrimiento se comparó con la clasificación especificada por la norma VDI 3198 [13]. 
El tratamiento térmico a $400^{\circ} \mathrm{C}$ desarrolló una buena adhesión entre el sustrato y el metal, sin desprendimiento de la capa de APQ ni formación de fisuras sobre la superficie como se observa en la Figura 1.
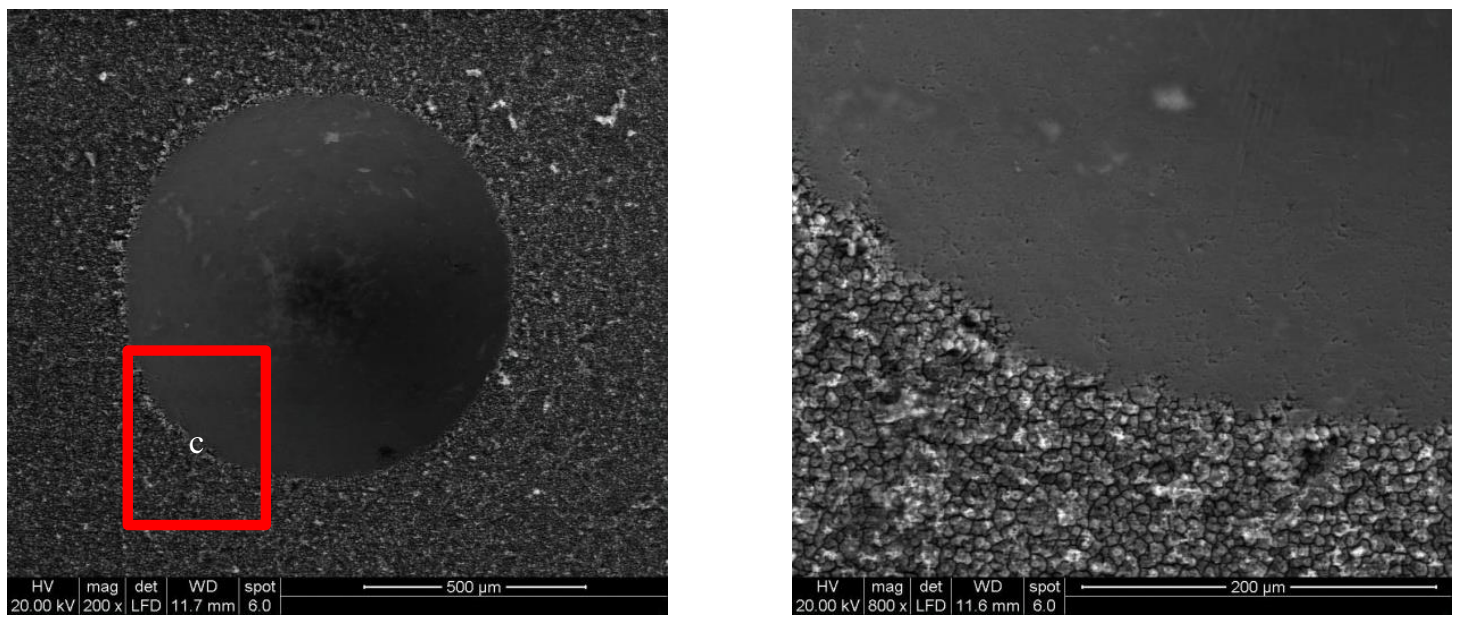

Figura 1:Imagen SEMde la indentación obtenida en el ensayo de adhesión de la muestra Blastinizado-APQ con tratamiento alcalino y térmico a $400{ }^{\circ} \mathrm{C}$, la imagen de la derecha representa el detalle de la indentación de la zona marcada en (a). La adhesión es evaluada como aceptable ya que el recubrimiento no presenta desprendimientos ni fisuras.

Las muestras tratadas a $600^{\circ} \mathrm{C}$ presentaron un comportamiento similar a las muestras tratadas a $400^{\circ} \mathrm{C}$, manifestando una buena adhesión entre el sustrato y el metal (ver Figura 2).
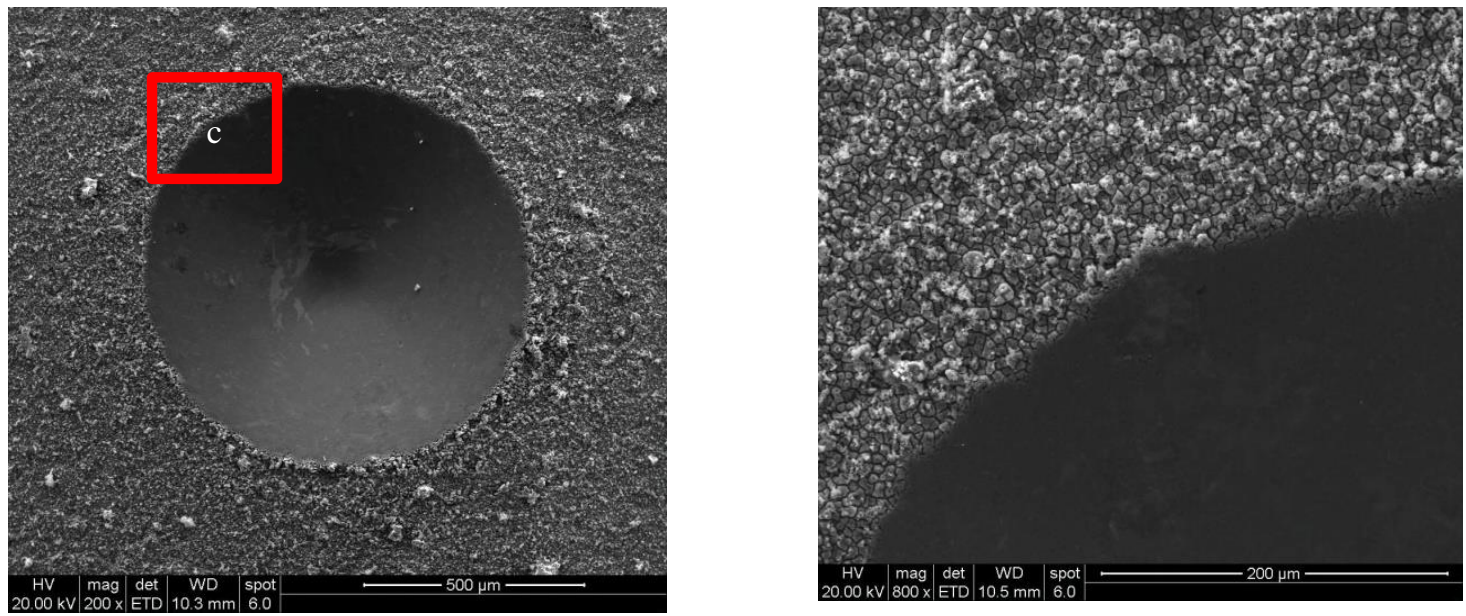

Figura 2: Imagen SEM de la indentación obtenida en el ensayo de adhesión de la muestra Blastinizado-APQ con tratamiento alcalino y térmico a $600{ }^{\circ} \mathrm{C}$, la imagen de la derecha representa el detalle de la indentación de la zona marcada en (a). La adhesión es evaluada como aceptable ya que el recubrimiento no presenta desprendimientos ni fisuras.

Las muestras tratadas a $800{ }^{\circ} \mathrm{C}$ presentaron una mala adhesión, en la Figura 3 se puede observar el desprendimiento del sustrato del metal. Este resultado se sustenta con los datos obtenidos en los ensayos de difracción de rayos x realizados a las muestras tratadas a $800{ }^{\circ} \mathrm{C}$ donde se observó una fase totalmente cristalina. 

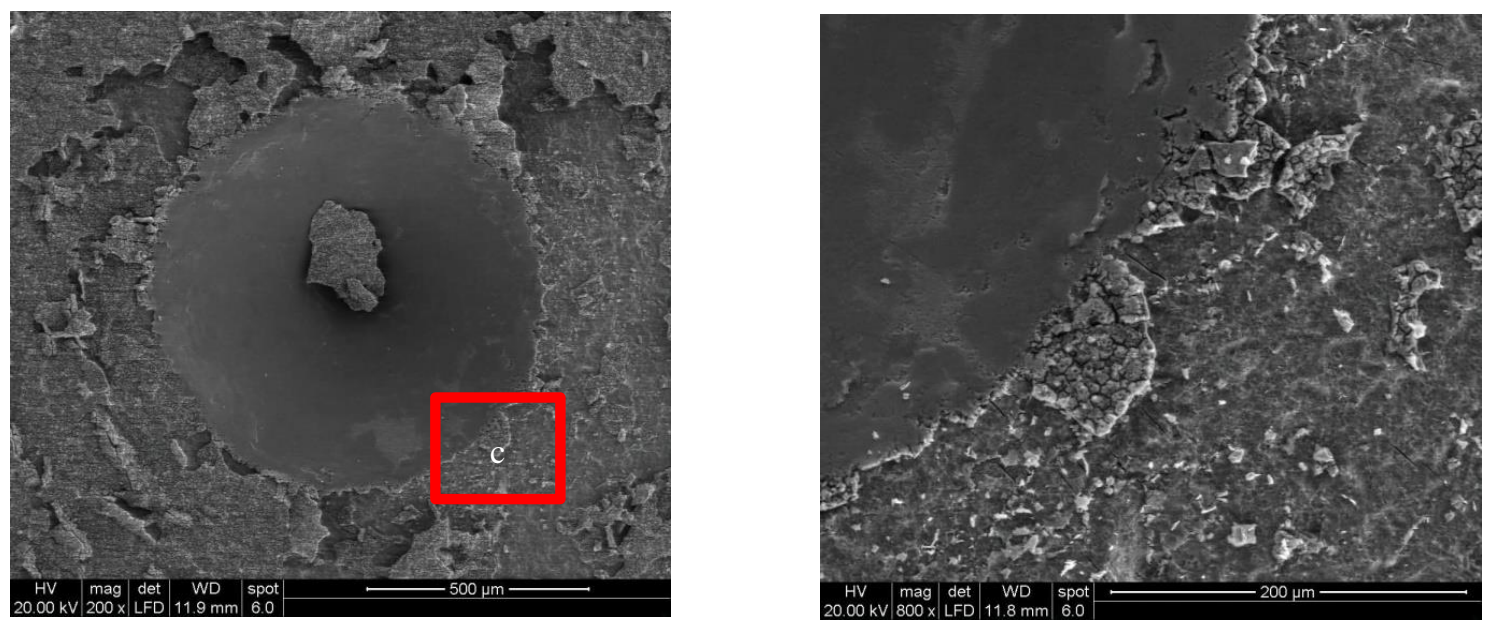

Figura 3: Imagen SEM de la indentación obtenida en el ensayo de adhesión de la muestra Blastinizado-APQ con tratamiento alcalino y térmico a $800{ }^{\circ} \mathrm{C}$, la imagen de la derecha representa el detalle de la indentación de la zona marcada en (a). La adhesión no es aceptable ya que presenta desprendimiento del recubrimiento y fisuras en la superficie.

\subsection{Difracción rayos $\mathrm{x}$}

El ensayo de difracción de rayos x permitió identificar la evolución de la fase amorfa a cristalina de la capa de titanato de sodio tras ser expuesta a tratamientos térmicos de distintas temperaturas.

Los tratamiento térmicos entre 400 y $800{ }^{\circ} \mathrm{C}$ aumentan la cristalinidad del recubrimiento. En la Figura 1 se pueden observar los difractogramas de muestras tratadas a 400,600 y $800{ }^{\circ} \mathrm{C}$ respectivamente.

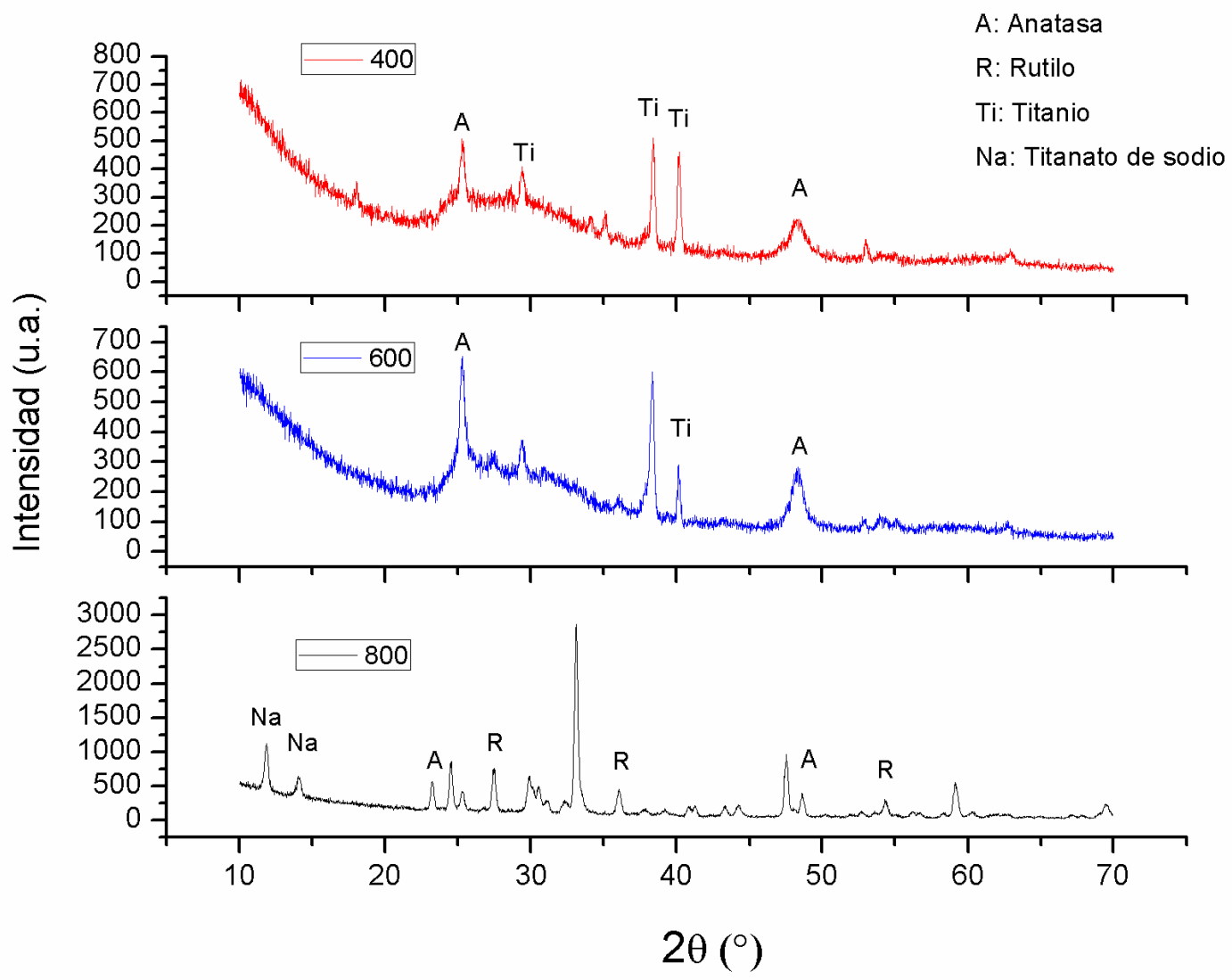

Figura 4: Difractogramas de las muestras con tratamiento térmico. Curva roja: $400^{\circ} \mathrm{C}$, azul: $600^{\circ} \mathrm{C}$, negra: $800^{\circ} \mathrm{C}$. A 
$400^{\circ} \mathrm{C}$ y $600^{\circ} \mathrm{C}$ se puede distinguir la presencia de una fase amorfa y a $800^{\circ} \mathrm{C}$ se pueden observar los picos de titanato de sodio, el cual cristalizo completamente.

En la curva de color rojo, correspondiente a la muestra tratada a $400^{\circ} \mathrm{C}$, se puede observar una zona de curvatura amorfa con picos anchos y de no muy alta intensidad. En la misma se identificaron picos compatibles con la presencia de fases cristalinas de titanio metálico como también de óxido de titanio correspondiente a la anatasa.

La curva que corresponde a la muestra tratada a $600^{\circ} \mathrm{C}$, de color azul, continúa revelando una fase amorfa. Se identificaron picos compatibles con la presencia de fases cristalinas de titanio metálico, al igual que de óxido de titanio correspondiente a la anatasa. Sin embargo hay algunas diferencias entre ambos difractogramas, como por ejemplo se puede observar un aumento en la intensidad de los picos de óxido de titanio (anatasa) en la muestra tratada a $600^{\circ} \mathrm{C}$ y a su vez una disminución de los picos del titanio metálico. A su vez en la curva azul los picos se observan más angostos y de mayor intensidad, manifestando de esta manera una mayor cristalización, en cambio los picos de la curva roja son más bien anchos.

Por último la curva de la muestra tratada a $800^{\circ} \mathrm{C}$ no presenta fase amorfa. Se identificaron picos compatibles con la presencia de fases cristalinas de anatasa y además la formación de rutilo. En este último difractograma también aparecen los picos característicos del titanato de sodio (Na2Ti6O13), que pudo ser detectado ya que cristalizo completamente.

\subsection{SBF}

Con el ensayo de inmersión en la solución de simulación de fluido humano (SBF) se evaluó la capacidad bioactiva de formar capas cerámicas de fosfato de calcio sobre sus superficies.

Las muestras fueron sumergidas durante 7 días a $37^{\circ} \mathrm{C}$. Tanto las probetas tratadas a 400 como a 600 ${ }^{\circ} \mathrm{C}$ desarrollaron la capa cerámica de fosfato de calcio. La caracterización de la capa formada se realizó con un microscopio electrónico de barrido, las figuras 5, 6,7 y 8 muestran la formación de una capa cerámica homogénea en toda la superficie de la muestra. La posterior caracterización analítica cualitativa se realizó con un espectrómetro dispersivo de energías que se puede observar en el apartado SEM-EDS.

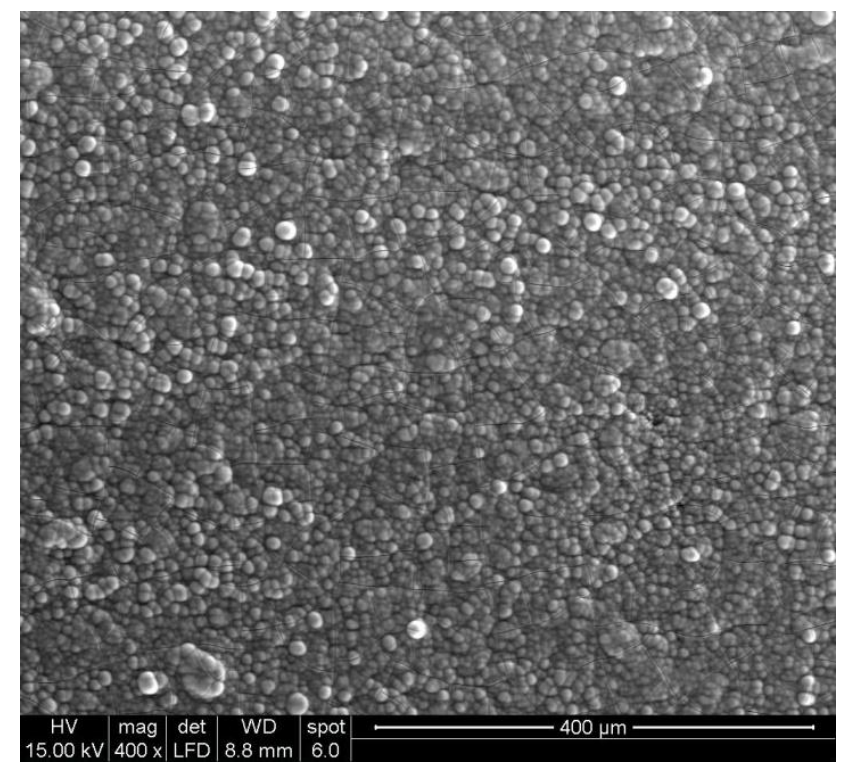

Figura 5: Imagen de la muestra $\mathrm{BAPQNaOH}-\mathrm{T} 400$ ensayada en SBF. La superficie se presenta totalmente cubierta por una capa de apatita luego de los 7 días de inmersión. 


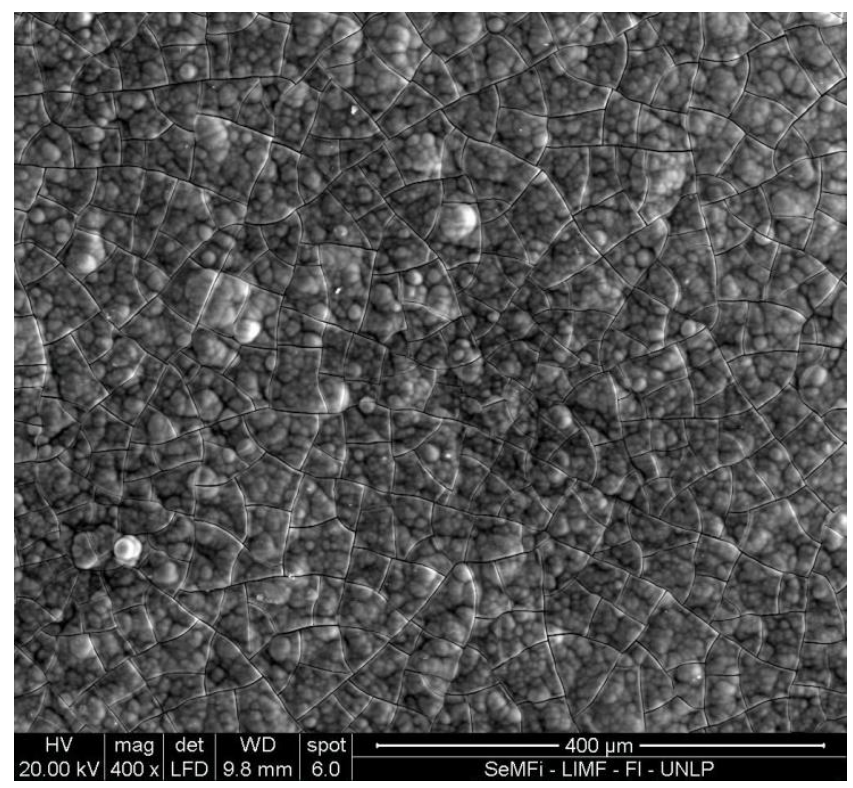

Figura 6: Imagen de la muestra $\mathrm{BNaOH}-\mathrm{T} 400$ ensayada en SBF. La superficie se presenta totalmente cubierta por una capa de apatita luego de los 7 días de inmersión.

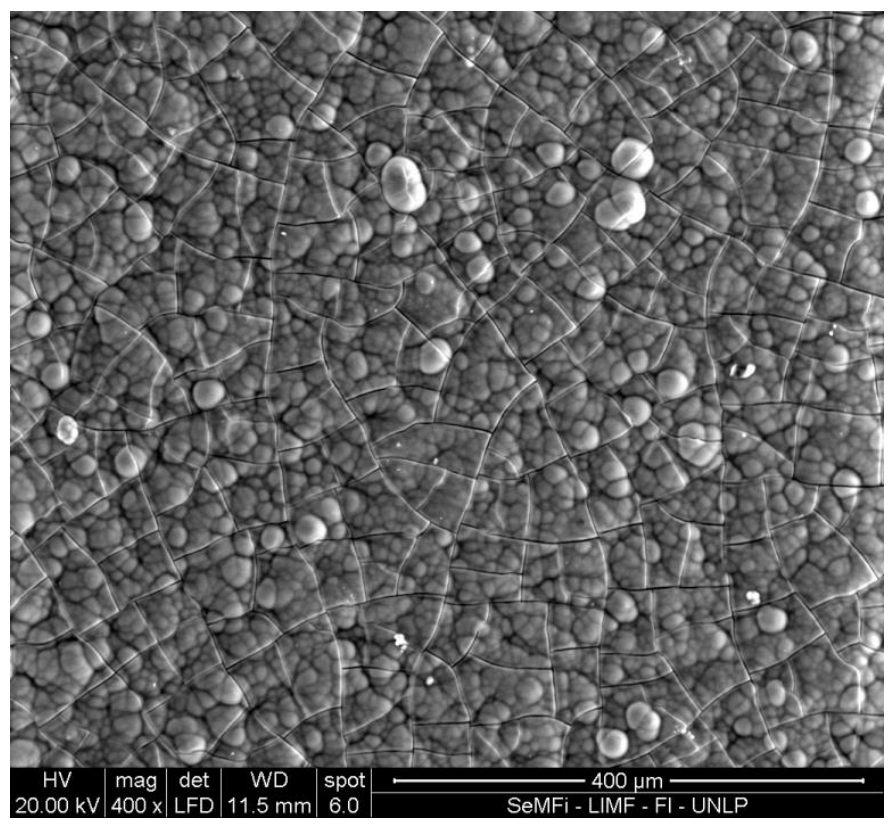

Figura 7: Imagen de la muestra BAPQNaOH-T600 ensayada en SBF. La superficie se presenta totalmente cubierta por una capa de apatita luego de los 7 días de inmersión. 


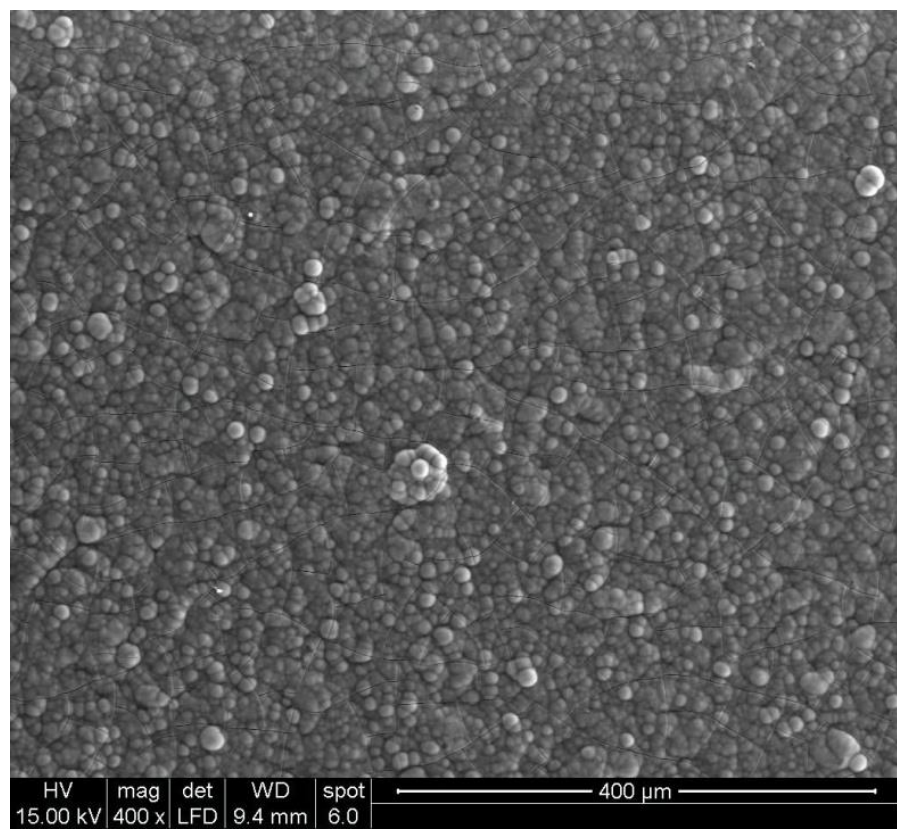

Figura 8: Imagen de la muestra $\mathrm{BNaOH}-\mathrm{T} 600$ ensayada en SBF. La superficie se presenta totalmente cubierta por una capa de apatita luego de los 7 días de inmersión.

Las muestras tratadas a $800^{\circ} \mathrm{C}$, luego de ser retiradas de la inmersión de SBF y secadas, se observaron en el microscopio electrónico de barrido y no presentaron ningún indicio de crecimiento de apatita sobre sus superficies durante los 7 días de ensayo. Este resultado se puede asociar con el alto porcentaje de cristalización que presentan los difractogramas de las muestras BAPQNaOH-T800 y BNaOH-T800, lo cual retarda el proceso de nucleación de la apatita [11].

\subsection{SEM-EDS}

Las muestras de blasting y APQ, tratadas alcalinamente y expuestas a temperaturas de 400 y $600^{\circ} \mathrm{C}$ desarrollaron en sus superficies la capa cerámica de fosfato de calcio. La misma se caracterizó cualitativamente mediante un espectrómetro dispersivo de energías (EDS) y se obtuvieron las relaciones de $\mathrm{Ca} / \mathrm{P}$ presentadas en la tabla 1 .

Tabla 3: Valor promedio de tres medidas de porcentaje atómico de Ca y P presente en las muestras luego de la inmersión en SBF.

\begin{tabular}{l|c}
\hline MUESTRAS & $\begin{array}{l}\text { PROMEDIO DE } \\
\text { \%AT CA/P }\end{array}$ \\
\hline BNaOH-T400 & 1,5 \\
\hline BAPQNaOH-T400 & 1,5 \\
\hline BNaOH-T600 & 1,5 \\
\hline BAPQNaOH-T600 & 1,5 \\
\hline
\end{tabular}

Estos resultados indican que en todas las superficies se forma el mismo compuesto correspondiente a una apatita ósea [12]. 


\section{DISCUSIÓN}

El tratamiento térmico realizado a las temperaturas de 400,600 y $800^{\circ} \mathrm{C}$ desarrollo en todas las muestras superficies muy hidrofilias con valores de ángulo de contacto muy bajos, comprendidos entre los $7 \mathrm{y} 11^{\circ}$.

Las muestras tratadas a $800{ }^{\circ} \mathrm{C}$ revelaron en su difractograma una fase completamente cristalina. Este resultado repercutió en los ensayos de adhesión, donde se observó desprendimiento de la capa de titanato y formación de fisuras, representando una adherencia inaceptable entre el recubrimiento y sustrato. Por otro lado, a pesar de desarrollar una superficie muy hidrofilica, en el ensayo de SBF se obtuvieron resultados negativos, ya que no se logró inducir el crecimiento de apatita sobre las superficies de las muestras tratadas durante el período de estudio. Este hecho se debe concretamente a que la formación de la apatita depende de la migración de iones de sodio provenientes del hidrogel de titanato de sodio que se forma sobre la superficie.

Si el hidrogel de titanato de sodio cristaliza, la liberación de los iones de sodio será mucho más lenta y retardará la nucleación de la apatita [11].

Las muestras tratadas a 400 y $600{ }^{\circ} \mathrm{C}$ manifestaron características muy similares entre sí. El difractograma de la muestra tratada a $400{ }^{\circ} \mathrm{C}$ revelo menor presencia de fase cristalina en comparación a la muestra tratada a $600{ }^{\circ} \mathrm{C}$. Estas diferencias se observaron en las dimensiones y la intensidad de los picos obtenidos en los difractogramas. Con respecto al ensayo de adhesión, ambas condiciones de temperatura respondieron favorablemente, representando una adherencia aceptable, sin desprendimientos del recubrimiento ni presencia de fisuras. Tras la inmersión de 7 días en SBF, las muestras desarrollaron superficies bioactivas que indujeron el crecimiento de apatita de un considerable espesor, ya que en los espectros realizados a altos voltajes de EDS $(25 \mathrm{kV})$ el porcentaje de titanio detectado es muy pequeño.

\section{CONCLUSIONES}

Tras analizar los resultados se concluye que la temperatura de $800{ }^{\circ} \mathrm{C}$ queda descartada como posible temperatura optima de trabajo ya que al cristalizar completamente retarda significativamente la nucleación de la apatita sobre la superficie de las muestras. Además representó una adherencia inaceptable entre el recubrimiento y el sustrato.

Las temperaturas de trabajo de 400 y $600{ }^{\circ} \mathrm{C}$ manifestaron características muy similares entre sí. Ambas desarrollaron superficies bioactivas que lograron nuclear la capa de fosfato de calcio y obtuvieron buena respuesta en cuanto a la adhesión del recubrimiento al sustrato. Para poder establecer una óptima condición de trabajo y seleccionar una sola temperatura, se deberá realizar un análisis cuantitativo, que consiste en medir el espesor de la capa cerámica de fosfato de calcio que se forma sobre una superficie y otra. Como también analizar la velocidad de nucleación de la capa de apatita en ambas condiciones. Del presente trabajo se concluye que las temperaturas de 400 y $600{ }^{\circ} \mathrm{C}$ son temperaturas que desarrollaron superficies hidrofilias, bioactivas y con buena adhesión.

\section{AGRADECIMIENTOS}

Los autores quieren expresar su agradecimiento a la empresa Kinetikal por el suministro de los materiales utilizados en este trabajo.

\section{BIBLIOGRAFÍA}

[1] ADYA, N., ALAM, M., RAVINDRANHAT, T., et al., "Corrosion in titanium dental implants: literature review", The journal of Indian Prosthodontic Society, v. 5, pp. 126-131, Jul.2015.

[2] ADDISON, O., DAVENPORT,A.J., NEWPORT,R.J., et al., "Do 'passive' medical titanium surfaces deteriorate in service in the absence of wear?", Journal of the Royal Society Interface, v. 9, n. 76, pp. 31613164, Jul. 2012.

[3] CARNEIRO-CAMPOS, L.E., FERNANDES, C.P., BALDUIÍNO, A., et al., "The effect of titanium topography features on mesenchymal human stromal cells' adhesion”, Clinical Oral Implants Research, v. 21, n. 2, pp. 250-254, Feb. 2010.

[4] YAN GUO, C., MATINLINNA, J.P., TANG,A.T.,"Effects of Surface Charges on Dental Implants: Past, Present, and Future",International Journal of Biomaterials, v. 2012, pp. 1-5,Sep. 2012.

[5] BRUSCHI, M., STEINMULLER-NETHL, D., GORIWODA, W., et al., "Composition and modifications of dental implant surfaces", Journal of oral implants, v. 2015, pp.1-14, Dec.2014.

[6] AGARWAL, A., TYAGI, A., AHUJA, A., et al., "Corrosion aspect of dental implants-An overview and 
literature review”, Open Journal of Stomatology, v. 4, n. 2, pp. 56-60, Feb. 2014.

[7] KRZĄKAŁA, A., KAZEK-KĘSIK, A., SIMKA,W., "Application of plasma electrolytic oxidation to bioactive surface formation on titanium and its alloys", RSC Advances, v. 3, pp. 19725-19743, Aug. 2013.

[8] LEMOS,A., CERLIANI,A., KANG,K., et al., "Caracterización superficial y transversal de implantes dentales de titanio blastinizados y anodizados por plasma químico". In: Actas del XI Congreso Iberoamericano de Ingeniería Mecánica CIBIM 2013, pp. 1108-1117, La Plata, Nov. 2013

[9] KIM, H.M., MIYAJI, F., KOKUBO, T., et al., "Effect of heat treatment on apatite-forming ability of Ti metal induced by alkali treatment", Journal of Materials Science Materials in Medicine,v. 8, n. 6,pp. 341-347, Jun. 1997.

[10] CUESTA,J., ESTUPIÑAN,H.A., PEÑA B., D.Y., et al., "Comportamiento electroquímico de recubrimientos Ca - P obtenidosmediante anodizado por plasma químico (APQ) tratados térmicamente", Scientia et Technica, v. 1, n. 36, pp. 233-238, Sep. 2007.

[11] APARICIO, C., PADRÓS, A., PLANELL, J.A., et al., "Superficies de titanio en implantes dentales de carga inmediata", Biomecánica, v. 14, n. 1, pp. 8-15, 2006.

[12] ZHAO, G., XIA, L., ZHONG, B., et al., "Effect of alkali treatments on apatite formation of microarcoxidized coating on titanium alloy surface", Transactions of Nonferrous Metals Society of China, v. 25, n. 4, pp. 1151-1157, Abr. 2015.

[13] VEREINDEUTSCHERINGENIEURE NORMEN, VDI 3198, VDI-Verlag, Dusseldorf, 1991.

[14] Implantsforsurgery - In vitro evaluationforapatite-formingability of implantmaterials, ISO 23317:2012.

[15] KOKUBO, T., MIYAJI, F., KIM, H.M., "Spontaneous formation of bonelike apatite laye ron chemically treated titanium metals", Journal of the American Ceramic Society, 79, n.4, pp.1127-29, 1996. 